Editorial

\title{
Macrolides in bronchiectasis
}

\section{Editorial}

Bronchiectasis is a chronic and etiologically heterogeneous inflammatory airway disease characterized by recurrent bacterial infection, irreversible dilatation of the bronchial walls, productive cough, and impaired quality of life. Recent epidemiological surveys revealed that the prevalence and the hospitalizations are steadily increasing globally although the true burden and the prevalence are still unknown. In the United States, trends in bronchiectasis diagnoses from 2000 to 2007 indicated annual percentage increase of $8.74 \%$ with the detection of 1,106 cases per 100,000 persons. Breaking the "vicious cycle" of infection, mucus stasis, inflammation, and airway destruction, including bolstering of immunity is the main therapy for bronchiectasis. Previous studies demonstrated that macrolides have considerable anti-inflammatory and immune-regulatory functions beyond their anti-microbial properties, particularly, 14- and 15 -membered ring macrolides. Their activities do not appear to be controlled by a single mechanism and have not yet to be elucidated. However, several previous studies demonstrated that macrolides down-regulate cytokine production by blocking the activation of the phosphorylation of extracellular single-regulated kinase 1/2 (ERK1/2) and the nuclear factor kappa B (NF-kappa B). Macrolides also mediate the innate and adaptive immune responses by inhibiting neutrophil activation. Macrolides have been effectively used in the treatment of chronic obstructive pulmonary disease, cystic fibrosis, and diffuse panbronchiolitis, but it remains uncertain how well can use in the management of non-cystic fibrosis bronchiectasis. Nevertheless, in patients with non-cystic fibrosis bronchiectasis, macrolide maintenance therapy can effectively decrease frequency of exacerbations, decrease sputum volume, attenuate lung function decline, and improve quality of life. Currently, apart from chest clearance techniques, effective long-term treatment are lacking. To decrease the growing clinical burden of this disease, the development of evidence-based therapy is urgently needed.
Volume 3 Issue 5 - 2016

\author{
Attapon Cheepsattayakorn, ${ }^{1,2,3}$ Ruangrong \\ Cheepsattayakorn ${ }^{4}$ \\ 'Editor-in-Chief, Journal of Lung, Pulmonary and Respiratory \\ Research, USA \\ ${ }^{2}$ IOth Zonal Tuberculosis and Chest Disease Center, Chiang Mai, \\ Thailand \\ ${ }^{3} 5$ th Office of Disease Prevention and Control, Ratchaburi, \\ Department of Disease Control, Ministry of Public Health, \\ Thailand \\ ${ }^{4}$ Department of Pathology, Faculty of Medicine, Chiang Mai \\ University, Chiang Mai, Thailand
}

Correspondence: Attapon Cheepsattayakorn, I0th Zonal Tuberculosis and Chest Disease Center, 143 Sridornchai Road Changklan Muang Chiang Mai 50I00 Thailand, Tel 6653 I40767, 6653 276364, Fax 6653 140773; 6653 273590,

Email Attapon1958@gmail.com, attaponche@yahoo.com

Received:September 17, 2016 | Published: September 19, 2016

\section{Acknowledgements}

None.

\section{Conflict of interest}

The author declares no conflict of interest. 